\title{
NRL S50T mutation and the importance of 'founder effects' in inherited retinal dystrophies
}

\author{
David AR Bessant ${ }^{1,2}$, Annette M Payne ${ }^{1}$, Catherine Plant ${ }^{2}$, Alan C Bird ${ }^{2}$, Anand Swaroop ${ }^{3,4}$ \\ and Shomi S Bhattacharya ${ }^{1}$
}

\footnotetext{
${ }^{1}$ D epartment of Molecular Genetics, Institute of Ophthalmology, London; ${ }^{2}$ M oorfields Eye Hospital, London, UK; Departments of ${ }^{3}$ Ophthalmology and ${ }^{4} \mathrm{H}$ uman Genetics, Kellogg Eye Centre, University of Michigan, Ann Arbor, MI, USA
}

The aim of this work was to identify NRL mutations in a panel of 200 autosomal dominant retinitis pigmentosa (adRP) families. All samples were subjected to heteroduplex analysis of the three exons of the NRL gene, and Hphl restriction digest analysis of exon 2 (to identify the S50T mutation). Families found to have the S50T mutation, and six additional larger pedigrees (which had previously been excluded from the other nine adRP loci) underwent linkage analysis using polymorphic markers located in the region of 14q11. Hphl restriction analysis followed by direct sequencing of the amplified NRL exon 2 product demonstrated the presence of the NRL S50T sequence change in three adRP families. Comparison of marker haplotypes in affected individuals from these families with those of affected members of the original 14q11 linked family revealed a common disease haplotype for markers within the adRP locus. Recombination events observed in these families define an adRP critical interval of $14.9 \mathrm{cM}$ between D13S72 and D14S1041. Linkage analysis enabled all six of the larger adRP pedigrees to be excluded from the 14q11 locus. The NRL S50T mutation represents another example of a 'founder effect' in a dominantly inherited retinal dystrophy. Identification of such 'founder effects' may greatly simplify diagnostic genetic screening and lead to better prognostic counselling. The exclusion of several adRP families from all ten adRP loci indicates that at least one further adRP locus remains to be found. European Journal of Human Genetics (2000) 8, 783-787.

Keywords: NRL; retinitis pigmentosa; retinal dystrophy; genetics; founder effect

\section{Introduction}

Retinitis pigmentosa (RP) (MIM 268000, Online Mendelian Inheritance in Man: http:www.ncbi.nIm.nih.gov/omim) is the term applied to a clinically and genetically heterogeneous group of retinal degenerations, which primarily affect the rod photoreceptors and have an overall prevalence of about 1:3000. ${ }^{1}$ RP is characterised by progressive loss of vision, initially manifesting a night blindness and reduction in the peripheral visual field, and later involving loss of central vision. ${ }^{2}$ Ophthalmoscopic examination typically

Correspondence: Professor Shomi S Bhattacharya, Department of Molecular Genetics, Institute of Ophthalmology, London, EC1V 9EL, UK. Tel: +44 207608 6806; Fax: +44 207608 6863; E-mail: sbhatach@hgmp.mrc.ac.uk

Received 6 August 1999; revised 22 May 2000; accepted 20 June 2000 reveals pigmentary disturbances of the mid-peripheral retina. RP may be inherited as an autosomal recessive, autosomal dominant, digenic, or X-linked trait. Autosomal dominant RP (adRP) accounts for $20-25 \%$ of all cases of RP. ${ }^{3}$

We recently reported an S50T mutation in the NRL gene, located at chromosome14q11, as the cause of adRP in a three-generation British family (designated RP251) linked to the marker D14S64 $\left(Z_{\max }=5.72\right.$ at $\left.\theta=0.00\right) .{ }^{4}$ adRP causing mutations have been identified in three other genes: rhodopsin (3q21-q25), ${ }^{5}$ peripherin-RDS (6p21), ${ }^{6}$ and RPI (8cen). ${ }^{7,8}$ In addition, six mapped adRP loci in which the responsible genetic mutation remains unknown are located at 1cen, $7 p$, 7q, 17p, 17q, and 19q (Retnet: http://www.sph.uth.tmc.edu/ Retnet/disease.ht).

We subsequently set out to screen a large panel of smaller adRP families for NRL mutations. 


\section{Patients and methods}

Two hundred apparently unrelated adRP families were identified from the Moorfields Eye Hospital retinal dystrophy register. The vast majority of these were British, originating from all over the country, but with the greatest number derived from the south and east due to the location of the hospital. After gaining informed consent, genomic DNA was prepared from peripheral blood obtained from members of these families. In order to identify NRL mutations all DNA samples were subjected to heteroduplex analysis and $\mathrm{Hphl}$ restriction digest analysis. In addition, six larger adRP pedigrees, which had previously been excluded from the other nine adRP loci, were analysed for linkage to markers within the RP251 critical region.

To aid heteroduplex detection new primers were synthesised from the genomic sequence of the gene, ${ }^{9}$ which produced a much smaller product from exon 1, and amplified both exon 2 and exon 3 in two parts (exon 1F - GATGACCTCAGAGAGCTGGC; 1R - GTTCTAGGTGAGCGGCCTGAC (216 bp); exon 2.1F - GGCCTCCATGTGCTCCAGAC; $2.1 \mathrm{R}$ GGCCTGGCCGGGTGCCCTCG (270 bp); exon 2.2F CCTTCAGTGAACCAGGCATGGT; 2.2R - CTCAGGCCAGCTTGCTGACC (256 bp); exon 3.1F CGGGTGCGACCTGGCGCTGAC; 3.1R - GCCAGGCGGGCCACCTCGGC (314 bp); exon $3.2 F$

GCCTGGCCGCCCAGCTGGAC; 3.2R - CCACTACACCACAAGGTGCTC (166 bp)). The resulting product was al lowed to cool slowly to room temperature to maximise the formation of heteroduplexes, ${ }^{10}$ and electrophoresed overnight at 1600-2000 Vh on MDE (Flowgen, Lichfield, Staffordshire, UK) polyacrylamide gels.

$\mathrm{Hphl}$ restriction digests were performed directly on $20 \mu \mathrm{l}$ of exon 2 PCR product by overnight incubation at $37^{\circ} \mathrm{C}$. Automated fluorescent sequencing ( $\mathrm{ABI}$ Biosystems, PerkinElmer Ltd, Beaconsfield, Buckinghamshire, (UK model 373)) of exon 2 of NRL was carried out using the above primers.

Those families found on sequencing to have an NRL S50T mutation underwent haplotype analysis using polymorphic markers in the region of the disease critical interval on $14 q 11$ (Table1). After PCR the amplified products were separated by electrophoresi s on 6-8\% non-den aturing polyacrylamide gels (Protogel, National Diagnostics, Atlanta, GA, USA/Hessle, Hull, UK).

All agarose and polyacrylamide gels were stained with ethidium bromide.

\section{Results}

The NRL S50T mutation is caused by a $T$ to $A$ change at nucleotide 1942 which abolishes a recognition site for $\mathrm{Hphl}$ restriction enzyme. $\mathrm{Hphl}$ restriction analysis followed by direct sequencing of the amplified NRL exon 2 product was therefore used to demonstrate the presence of this sequence change in the NRL gene in three adRP patients from familiesRP57, RP357, and RP3097 (Figure1). No other sequence changes were detected in any exon of the NRL gene in any patient sample.

Comparison of marker haplotypes in affected individuals from familiesRP57 and RP357 with those of affected members of the original RP251 family revealed a common disease haplotype for markers within the adRP locus on $14 q 11$ (Table1). The same disease haplotype can be inferred from the alleles present in individual number 1727 from family RP3097, but since only one member of this family was analysed this cannot be confirmed. This disease-associated haplotype was not observed on any of the 16 'control' chromosomes, belonging to individuals who were related by marriage to these families (RP57, RP251, RP357 and RP3097), which underwent haplotyping. Rare disease-associated alleles of both D14S990 (allele3) and D14S64 (allele5) were found to occur at a significantly higher frequency in the affected individuals from these families. $\chi^{2}$ : $P=0.003$ for each marker).

Recombination events in affected individuals from the original family RP251 define a $17 \mathrm{cM}$ critical genetic interval for this locus between the markersD14S261 and D14S1041 (Figure2). A recombination event observed in subject number 87 , from family RP357, reduces the adRP critical interval to $14.9 \mathrm{cM}$ and defines D14S72 as the new centromeric boundary (Table1).

Table 1 Comparison of the suggested haplotypes of polymorphic markers in the critical region on $14 q 11$ between RP251 and three additional families with the codon 50 Ser to Thr mutation in the NRL gene identified by restriction digest analysis. The presence of the same variant allele for the centromeric marker, D14S261, in all additional cases may indicate a recombination event in the founder of RP251. Subject No. 87 has a recombination involving D14S 72 which refines the adRP critical interval to 14.9cM. Marker order obtained from Dib et $\mathrm{al}^{19}$

\begin{tabular}{|c|c|c|c|c|c|c|c|c|c|c|c|}
\hline \multirow{3}{*}{$\frac{\text { Marker }}{\text { D14S } 261}$} & \multirow{3}{*}{$\begin{array}{c}\text { RP251 } \\
\text { Haplotype } \\
2\end{array}$} & \multicolumn{4}{|c|}{ RP57 } & \multirow{2}{*}{\multicolumn{2}{|c|}{$\begin{array}{c}\text { RP3097 } \\
\text { No. } 1727\end{array}$}} & \multicolumn{4}{|c|}{ RP357 } \\
\hline & & \multicolumn{2}{|c|}{ No. 2503} & \multicolumn{2}{|c|}{ No. 2504} & & & \multicolumn{2}{|c|}{ No. 2399} & \multicolumn{2}{|c|}{ No. 87} \\
\hline & & 4 & 1 & 4 & 5 & 4 & 4 & 4 & 4 & 4 & 5 \\
\hline D14S 72 & 2 & 2 & 1 & 2 & 4 & 2 & 4 & 2 & 3 & 4 & 5 \\
\hline D14S 990 & 3 & 3 & 2 & 3 & 2 & 3 & 4 & 3 & 1 & 3 & 3 \\
\hline D14S 64ª & 5 & 5 & 3 & 5 & 4 & 5 & 1 & 5 & 2 & 5 & 3 \\
\hline D14S 1041 & 2 & 2 & 1 & 2 & 4 & 2 & 4 & 2 & 1 & 2 & 4 \\
\hline D14S 80 & 3 & 3 & 3 & 3 & 4 & 3 & 5 & 3 & 3 & 3 & 3 \\
\hline
\end{tabular}

aLocation of NRL; Farjo et al ${ }^{9}$ 


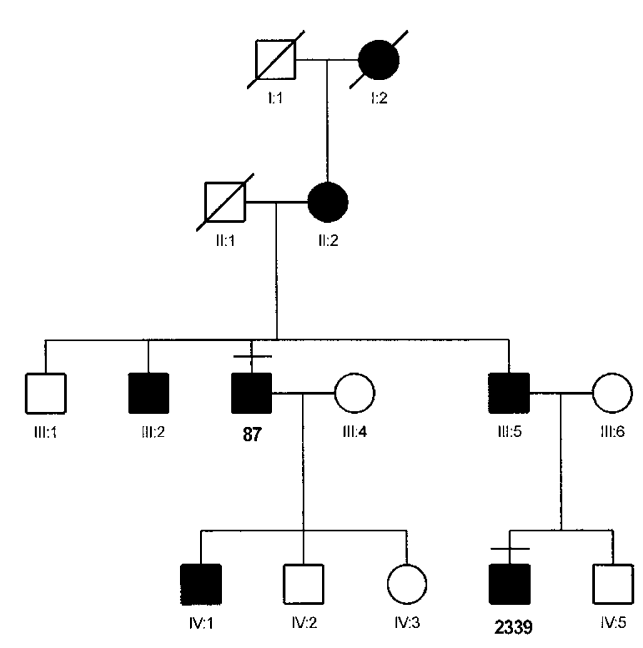

RP357

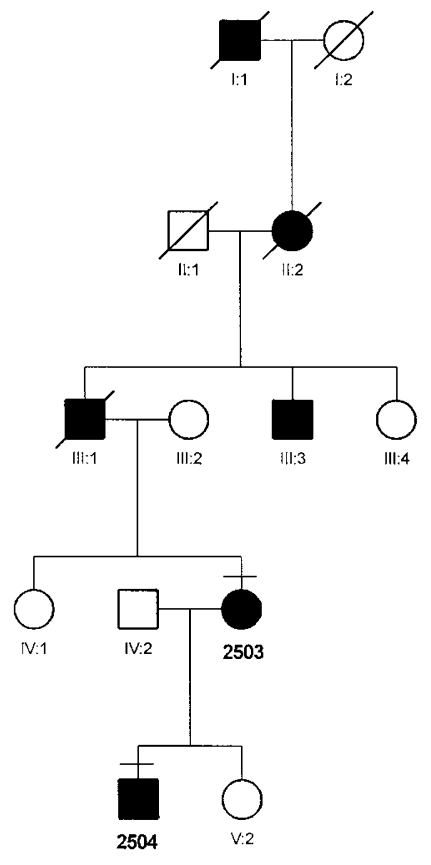

RP57

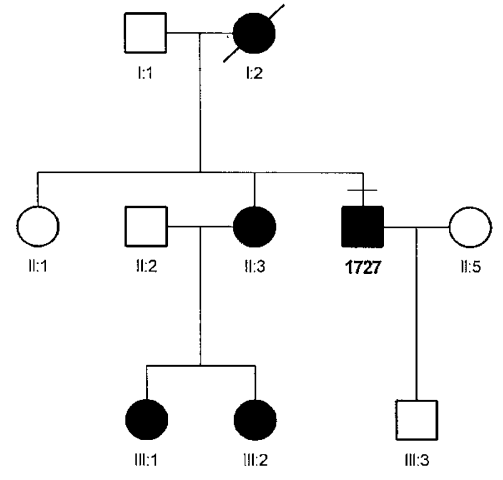

RP3097

Figure 1 Pedigrees of additional families with the NRL S50T mutation. Patients whose DNA samples underwent mutation and haplotype analysis are denoted by a horizontal bar above the symbol representing that individual. DNA numbers correspond with those in Table1 (affected individuals are denoted by solid symbols).

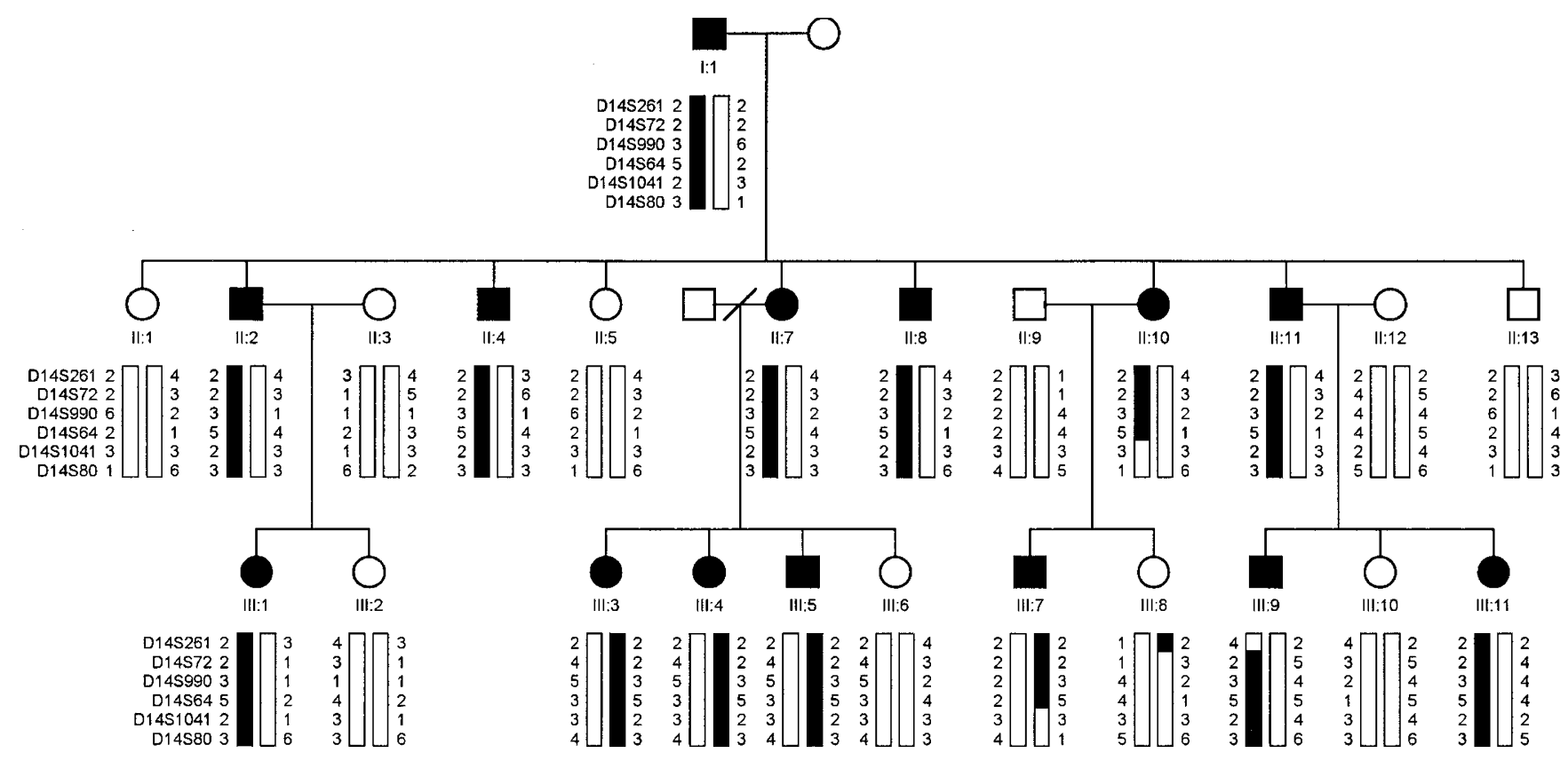

Figure 2 Pedigree of family RP251 showing haplotypes for the polymorphic markers in the centromeric region of chromosome14q. Marker order was determined from the Généthon sex-averaged genetic map. ${ }^{19}$ Centromeric recombination events in individuals III-8 and III-9, and a telomeric recombination in affected individual II-10 define D14S261 and D14S1041 as the flanking markers for the adRP locus. The affected haplotype is indicated by solid black in the bars situated between the marker alleles. 
Linkage analysis enabled all six of the larger adRP pedigrees to be excluded from the $14 q 11$ locus (data not shown).

\section{Discussion}

The identification of a tenth locus for adRP at $14 q 11$ further highlights the genetic heterogeneity which is associated with this condition and with non-syndromic retinal dystrophies in general (RetNet). The exclusion of additional adRP families from all ten adRP loci indicates that at least one further adRP locus remains to be found.

Since affected individuals from these four families (RP57, RP251, RP357, and RP3097) share nearly $15 \mathrm{cM}$ of chromosome $14 q$ surrounding the NRL gene it seems likely that they are, in fact, closely related and that the NRL S50T mutation arose relatively recently. The earliest known affected family member from these pedigrees (from RP57) was born around 1850. These four families all originate from south-east England and genealogical studies are in progress to determine the exact relationship between them.

In all four pedigrees the RP phenotype is fully penetrant and exhibits only limited variation in expressivity. This is comparable with the phenotypes ascribed to specific rhodopsin mutations and in contrast to the variable expressivity and incomplete penetrance associated with the $7 p$, 8cen and $19 q$ adRP loci respectively. ${ }^{11-13}$ Detailed, clinical, electrophysiological and psychophysical studies of affected individuals from these four families suggest that there may be characteristic features of the 14q11 adRP phenotype (including early severe loss of rod function, whilst cone function is preserved and a very high incidence of macular oedema), which may aid in the identification of additional linked families (Bessant et al., manuscript in preparation).

Since we have only been able to link one adRP pedigree to the $14 q 11$ locus, and since mutation screening of NRL in 200 additional adRP families revealed only three additional, related families it seems likely that mutations at this locus are a relatively rare cause of adRP. The fact that the additional three pedigrees are related to the family RP251 is further evidence of the importance of the 'founder effect' in autosomal dominant retinal dystrophies. There now exist several striking examples of this effect:

(1) the rhodopsin Pro23His mutation which accounts for around $10 \%$ of cases of dominant retinitis pigmentosa in the United States; ${ }^{14}$

(2) the TIMP-3 Ser181Cys mutation in patients of British origin with Sorsby fundus dystrophy; ${ }^{15}$

(3) the peripherin-RDS Arg172Trp mutation in dominant macular dystrophy; ${ }^{16}$

(4) the many descendants of Jean Nougaret with congenital stationary night blindness due to a mutation in GNAT $1 ;{ }^{17}$ and
(5) perhaps most spectacularly the 39 apparently unrelated pedigrees from several different countries with dominant drusen of the Doyne/Malattia leventinese type, all of whom were found to have an Arg345Trp mutation in the gene EFEMP1. ${ }^{18}$

Identification of 'founder effects' in local populations, or in some cases internationally, can greatly simplify genetic analysis of the relevant disease and may have important implications for the speed and cost-efficiency of diagnostic screening services. Associated clinical studies may provide accurate information about disease progression for the purpose of prognostic counselling. Large groups of individuals whose eye disorder is due to a single mutation are also ideal candidates for recruitment into trials of any potential future treatment for the inherited retinal dystrophies.

\section{Acknowledgements}

We would like to thank the family members for their participation in this study. David Bessant and Annette Payne are supported by the Medical Research Council of the UK (Grant No.G9301094). AS is supported by grants from the National Institutes of Health (EY11115) and the Foundation Fighting Blindness. SSB would like to acknowledge the support of the Foundation Fighting Blindness and the Ulverscroft Foundation. We would also like to thank the Wellcome Trust for an Equipment Grant for the sequencing facility (No.039283/2/93/Z/MW/JF). DARB and AMP have contributed equally to this work.

\section{References}

1 Bundey S, Crews SJ: A study of retinitis pigmentosa in the city of Birmingham. I. Prevalence. J Med Genet 1984; 21: 417-420.

2 Bird AC: Retinal photoreceptor dystrophies. Am J Ophthalmol 1995; 119: 543-562.

3 Jay $\mathrm{M}$ : On the heredity of retinitis pigmentosa. $\mathrm{Br}$ J Ophthalmol 1982; 66: 405-416.

4 Bessant DAR, Payne AM, Mitton KP et al: A mutation in NRL is associated with autosomal dominant retinitis pigmentosa. $N$ at Genet 1999; 21: 355-356.

5 Gal A, Apfelstedt-Sylla E, Janecke A, Zrenner E: Rhodopsin mutations in inherited retinal dystrophies and dysfunctions. Prog Retinal Eye Res 1997; 1: 51-79.

6 Keen TJ, Inglehearn CF: Mutations and polymorphisms in the human peripherin-RDS gene and their involvement in inherited retinal degeneration. Hum Mutat 1996; 8: 297-303.

7 Pierce EA, Quinn T, Meehan T, McGee TL, Berson EL, Dryja TP: Mutations in a gene encoding a new oxygen-regulated photoreceptor protein cause dominant retinitis pigmentosa. Nat Genet 1999; 22: 248-254.

8 Sullivan LS, Heckenlively JR, Bowne SJ et al: Mutations in a novel retina-specific gene cause autosomal dominant retinitis pigmentosa. Nat Genet 1999; 22: 255-259.

9 Farjo $\mathrm{Q}$, Jackson A, Pieke-Dahl S et al: Human bZIP transcription factor gene NRL: Structure, genomic sequence and fine linkage mapping at $14 q 11.2$ and negative mutation analysis in patients with retinal degeneration. Genomics 1997; 45: 395-401.

10 Keen J, Lester D, Inglehearn CF, Curtis A, Bhattacharya SS: Rapid determination of single base pair mismatches as heteroduplexes on Hydrolink gels. Trends Genet 1991; 7: 5.

11 Inglehearn CF, Carter SA, Keen TJ et al: A new locus for autosomal dominant retinitis pigmentosa (adRP) on chromosome7p. Nat Genet 1993; 4: 51-53. 
12 Xu S-Y, Denton M, Sullivan LS, Daiger SP, Gal A: Genetic mapping of RP1 on 8q11-q21 in an Australian family with autosomal dominant retinitis pigmentosa reduces the critical region to $4 \mathrm{cM}$ between D8S601 and D8S285. Hum Genet 1996; 98: 741-743.

13 Al-Maghtheh $M$, Inglehearn CF, Keen TJ et al: Identification of a sixth locus for autosomal dominant retinitis pigmentosa on chromosome19. Hum Mol Genet 1994; 3: 351-354.

14 Dryja TP, Hahn LB, McGee TL, Cowley GS, Berson EL: Mutation spectrum of the rhodopsin gene in patients with autosomal dominant retinitis pigmentosa. Proc Natl Acad Sci USA 1991; 88: 9370-9374.

15 Wijesuriya SD, Evans K, Jay MR et al: Sorsby's fundus dystrophy in the British Isles: demonstration of a striking founder effect by microsatellite-generated haplotypes. Genome Res 1996; 6: 92-101.
16 Payne AM, Downes SM, Bessant DAR, Bird AC, Bhattacharya SS: Founder effect seen in the British population of the 172 peripherin/RDS mutation and further refinement of genetic positioning of the peripherin/RDS gene. Am J Hum Genet 1998; 62: 192-195.

17 Dryja TP, Hahn LB, Reboul T, Arnaud B: Missense mutation in the gene encoding the alpha subunit of rod transduction in the Nougaret form of congenital stationary night blindness. N at Genet 1996; 13: 358-360.

18 Stone EM, Lotery AJ, Munier FL et al: A single EFEMP1 mutation associated with both Malattia Leventinese and Doyne honeycomb retinal dystrophy. Nat Genet 1999; 22: 199-202.

19 Dib C, Faure S, Fizames C et al: A comprehensive genetic map of the human genome based on 5,264 microsatellites. Nature 1996; 380: 152-154. 